\title{
An Online Fitting Simulation System of a Garment Using 3D Body Data
}

\author{
Ran Choi*, Chang-Suk CHO* \\ "School of IT, Hanshin University, Yangsan-dong, Osan-si, Korea;
}

\begin{abstract}
We propose a garment fitting system for an online retail model, which uses a consumer's 3Dbody data for garment fitting. The front and back images of a garment is modeled as a garment to be fitted and the 3D laser scanned body data is modeled as the body. This system was made to imitate the way how human fits a garment, such as fitting a top from his neck to downwards and fits pants upwards. In order to do it, a simulation process for combining the 3D data and garment images is reported, which includes modeling, collision detection and feedback handling. As inputs of the garment fitting system, One-piece, T-shirts and pants were tested and reported with its successful results in this system. The resulting system would support an online retail business model for garments. In the proposed business model, a body data center would scan the body and store the 3D data, the simulated garment fitting program would be provided to individual consumers for fitting on a home PC, and retail shops would exhibit garment images on their websites.
\end{abstract}

Keywords: 3D body, laser scan, fitting simulation, garment

\section{Introduction}

Currently, online clothing store websites typically only display 2D images of the garments that they offer for sale. They offer no user-interaction or feedback regarding the fit of the garment for a particular user's body type and size. This paper presents a 3D interactive simulated garment-fitting system that uses 3D laser scanned body data and 2D garment images. The proposed system introduces a new garment fitting approach based on the way how human fits a garment, such as fitting a top from his neck to downwards and fits pants upwards. The resulting system would support an online retail business model for garments. In the proposed business model, a body data center would scan the body and store the 3D data, the simulated garment fitting program would be provided to individual consumers for fitting on a home PC, and retail shops would exhibit garment images on their websites. It is important for a simulated garment-fitting system to accurately combine 3D body data and 2D garment images. This requires the geometrical and physical modeling of clothes, collision detection between body data and clothes, and also feedback handling techniques. There are several existing approaches to simulated garment fitting. Seo et al. present a cloth simulator for estimating pressure from tightly fitted clothing, in which physical measurements of pressure were compared to simulated pressure estimates [1]. Magnenat-Thalmann et al. used an avatar model for garment fitting, deforming an avatar to the body size of the consumer [2]. Cordier et al. presented a well-designed online clothing retail system that uses a web application to manipulate garment related items, facilitating clothing design, pattern derivation and sizing [3]. But these systems use avatar model or require the information on the composition of patterns. Whereas the proposed system uses the 3D laser scanned body data instead of using an avatar and was made to imitate the way how human fits a garment, such as fitting a top from his neck to downwards and fits pants upwards. Cho et al. also reported the online fitting system using the 3D laser scanned body data and the imitation of fitting process of human [4]. We enhanced the system, reported in [4], to modify its physical modeling and to introduce fitting the top and bottom in layers. From section 2 the enhanced system is reported.

\section{Fitting process of a garment}

\subsection{D body dataset}

We used 3D body dataset scanned by the Cyberware Whole Body Scanner as 3D model of the customer's body [4]. One hundred and four female bodies, whose ages are about twenty, were scanned to collect the 3D body dataset. This body dataset is composed of about 120,000 points and the 260,000 polygons defined from these points. After scanning, the body data is transformed to ASE (ASCII Standard Export) file format, which consists of 3D coordinate position vectors as shown in Figure 2, connection information among the vectors, and textures. The ASE dataset is about $60 \mathrm{MB}$ per person and its resolution is approximately within $1 \mathrm{~mm}$ in both the horizontal and vertical axes.

* cscho@hs.ac.kr; +1-82-31-379-0652; cglab.hs.ac.kr 


\subsection{Virtual fitting process for garment}

\subsubsection{Modeling of $2 D$ garment image}

2D image of a garment is extracted based on 22 points preliminarily determined using the image tool in figure 1. We made a simple tool for extracting garment images, which extracts front and back images by fitting lines manually to the edges of the garments in the images. Figure 1 shows the edge extracting tool. The point $\mathrm{Cp}$ in figure 1 is used as a base point for the merging process, which corresponds to the front neck point of the body. $\mathrm{Cp}$ is determined as the centroid between P1 and P2 in figure 1. Front and back images of clothes are used for the fitting simulation, and the length of the garment is recorded as a basis for fitting. The backgrounds of the two images are removed in order to extract the garment image, which is then mapped onto a polygon mesh for the fitting process.

Also the points from e1 to e8 in figure 1 are used as reference points to determine whether the fitting is finished or not. In order to determine finishing condition of the top the shoulder-sleeve points are used as the references and the edge points of pants are used for finishing condition of the bottom.
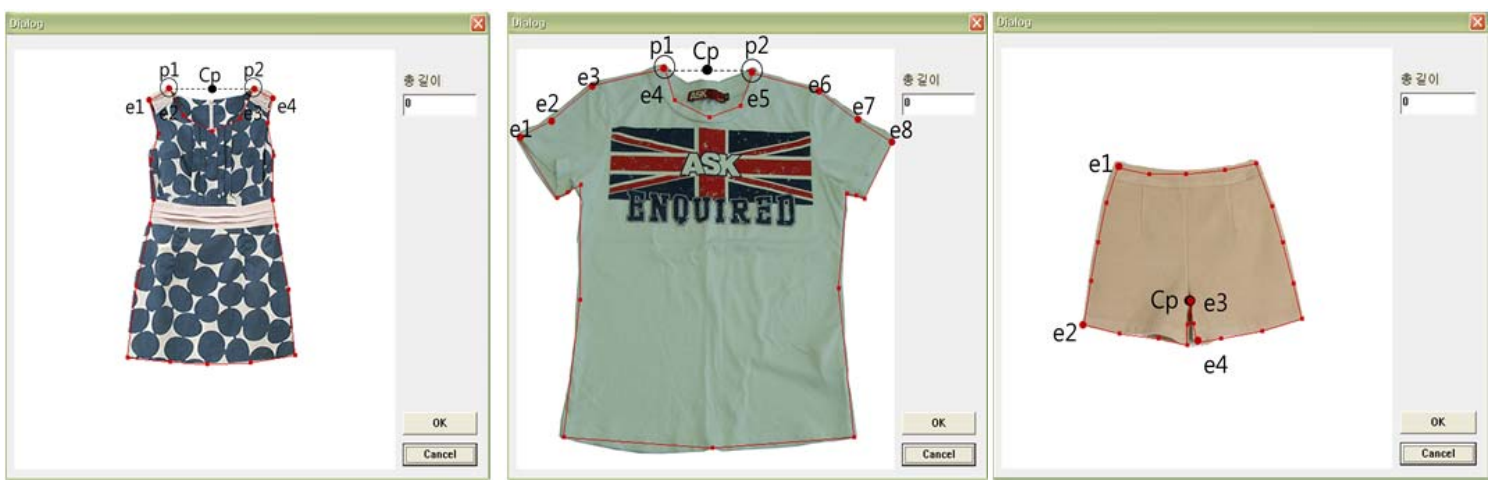

Fig. 1. Extracting front and back images of garment and determining the base point for fitting.

To fit the garment images from front and back on the body data, the structural and physical characteristics of the cloth must be applied to the images. There were some researches related to the modeling of the images. Haumann first introduced a mass-spring model for virtual garments, in which each side of a triangle was treated as a spring and each vertex as a mass point $[5,6]$. Provot also proposed a lattice style spring model in which structural and sheer springs were used [7]. In our research, a tension spring in a mass-spring model based on triangles is used, as seen in figure 2

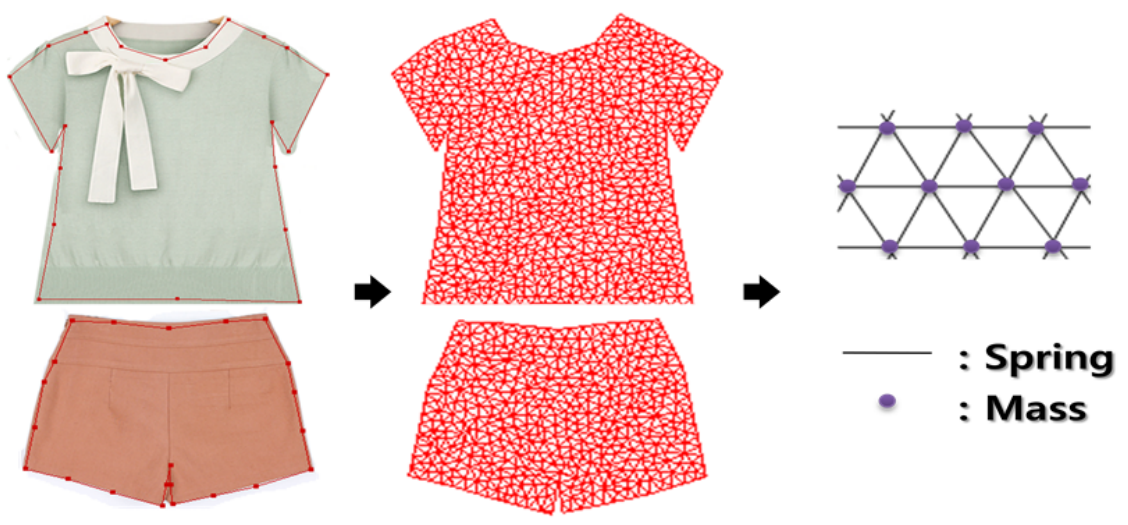

Fig. 2. Mass-spring model for a garment image.

Shear, structural spring models are needed when considering various clothing styles and dynamic situations, such as the wearer walking or windy conditions, whereas only tension springs are used here, as we assume a static situation. A force applied to the springs is defined in equation 1 where the force is proportional to the distance between particles and applied to the spring as a damping power resisting the relative velocity of the particle [5]. 


$$
\begin{gathered}
f_{i}^{s}=-k_{i j}\left(\left|\Delta P_{i j}-l_{i j}\right|\right)\left(\frac{\Delta P_{i j}}{\left|\Delta P_{i j}\right|}\right)-k_{i j}^{d} \Delta V_{i j}\left(\frac{\Delta P_{i j}}{\left|\Delta P_{i j}\right|}\right) \\
\Delta P_{i j}=P_{i}-P_{j}, \quad \Delta V_{i j}=V_{i}-V_{j}
\end{gathered}
$$

In Equation $1, P_{i}$ denotes the position vector of particle i in $x-y-z$ coordinates, $V_{i}$ the velocity vector of particle $i, K_{i j}$ the spring constant for the spring between particles $i$ and $\mathrm{j}, k_{i j}^{d}$ the damping constant of the spring, $l_{i j}$ the original distance between particles, and $f_{i}^{s}$ tension vector of the spring on particle $i$.

We do not require dynamic posture in the fitting simulation, so viscosity and wind effects on the cloth are omitted here. But even in the static state, friction and gravity affect cloth. Therefore, we added frictional and gravitational forces to the model. When putting on a top, the front neck point is used as a base, and putting on a bottom the groin done.

\subsubsection{Fitting process of a top}

Under the assumption that cloth is a structure composed of connected particles, a mass-spring model is constructed that describes the cloth shape and its deformation from interactions with other particles. The front and back garment images are merged dynamically and stitched together, positioning the body between the two images. To embody this dynamic process of merging, the variation of position over time is computed using an energy equation. Equation 2 shows the calculation of a particle position over time using Explicit Euler Integration [8].

$$
\begin{aligned}
& V_{n+1}=V_{n}+F_{n+1} d t / m \\
& P_{n+1}=P_{n}+V_{n+1} d t
\end{aligned}
$$

In equation 2, $P$ denotes the position vector of a particle, $V$ the velocity vector of a particle, $F$ a force vector applied to a particle, $m$ the mass of the particle, and $n$ is the time step. Using these equations, the two images are merged. The force worked to cloth before stitching includes the tension power of the spring in equation 1, the stitching power between the front and back images of cloth, and the friction power between the body and cloth, which is shown in equation 3.

$$
F_{i}=f_{i}^{s}+f_{i}^{a}+f_{i}^{f}
$$

$f_{i}^{S}$ is the tension vector of the spring on particle $i$ as shown in equation $1, f_{i}^{a}$ the stitching power between the front and back images of cloth and $f_{i}^{f}$ and the friction power between the body and cloth. The stitching power works between the front and back images of cloth, so that the two images can be merged and stitched by the power. The friction power works between the cloth and the body, so that it helps for the cloth to keep its position from preventing rolling down. The friction power of a particle i can be computed in equation 4 , and the $\mu$ and $V_{i}$ in the equation represent the friction coefficient and speed vector of the particle respectively. The friction power is applied along $x-z$ coordinates only to the particle which was detected having collision. The friction coefficient here was set to -0.01 , which was determined empirically.

$$
f_{i}^{f}=\mu V_{i}
$$

After stitching, the stitching power in equation 3 is removed and the gravity is added newly, so that the new power $F_{i}$ is composed of the tension $f_{i}^{s}$, the friction $f_{i}^{f}$ and the gravity $f_{i}^{g}$ as shown in equation 5 . In the equation $m_{i}$ is the mass of a particle $i, G$ the acceleration vector of gravity.

$$
\begin{aligned}
& F_{i}=f_{i}^{s}+f_{i}^{f}+f_{i}^{g} \\
& f_{i}^{g}=m_{i} G
\end{aligned}
$$

The particles of cloth are controlled by the interaction of the powers and collide with the polygons on the body. To detect the collision we divided the body spatially into sub-cell voxels $[3,5]$. The polygons on the body surface must be registered to a cubic space composed of voxels. In order to do this, the body is inscribed into a rectangular parallelepiped cell which includes all polygons of the body surface, 
and the rectangular parallelepiped is divided into sub-cell voxels, whose side length is 0.5 pixel. After the division, each polygon on the body surface is registered to the corresponding voxel.

The collision between a particle of cloth and a polygon of the body is regarded as an inelastic collision, since the fitting in this research is assumed as standing posture without moving. When a particle of cloth collides with a polygon of the body to a velocity $V$, the new velocity $V^{\prime}$ after the collision is determined by the impulse at the collision. Since inelastic collision is assumed here, the velocity $V$ ' has only horizontal component in its direction and the vertical component is removed.

To finish the fitting process of the top a finishing condition is designed using shoulder-sleeve points. The shoulder-sleeve points are of 2 or 4 points per a shoulder from the initial part to the end part of shoulder as shown in figure 1, in which the one-piece has two shoulder-sleeve points for the finishing condition but T-shirt has four points as the finishing condition. Equation 6 shows the finishing condition. In the equation, $P_{i}$ is the position vector on a particle i of cloth, $F$ the power vector, $V$ the velocity vector and $N$ the normal vector.

$$
\begin{array}{ll}
I F(|\Delta d|<\epsilon \mid \epsilon \rightarrow 0) & \left\{\begin{array}{cl}
\text { Yes, } & V_{n+1}=0 \\
\text { No, } & V_{n+1}=V_{n}+F_{n+1} d t / m
\end{array}\right. \\
\Delta d=N-P_{i} &
\end{array}
$$

Figure 3 shows the fitting process for a top whose lines across the body represent the stitching power vector between the front and back images of cloth.
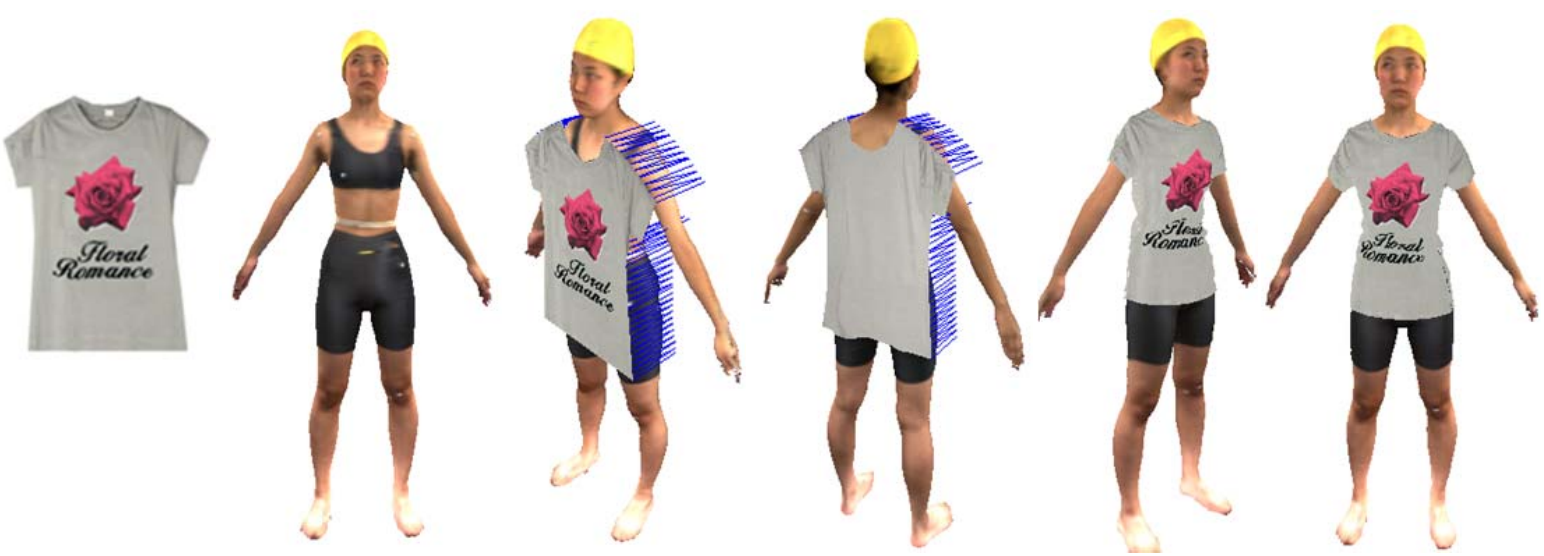

Fig. 3. The fitting process for a top.

\subsubsection{Fitting process of a bottom}

When fitting a bottom to the body, the groin point is the base for fitting. We set the initial position of the front and back images of the bottom to the base point and put the bottom on the body merging and stitching as same process as the top and pulling up it on the contrary to the top. To pull up the bottom, antigravity force is introduced. Equation 7 shows the fitting process for pulling up the bottom after stitching.

$$
\begin{array}{ll}
I F(|\Delta d|<\epsilon \mid \epsilon \rightarrow 0) & \begin{cases}\text { Yes, } & V_{n+1}=0 \\
\text { No, } & V_{n+1}=V_{n}+F_{n+1} d t / m+f_{i}^{f}\end{cases} \\
\Delta d=C_{p}-p_{p}
\end{array}
$$

In equation $7, C_{p}$ is the crotch of the bottom and $P_{p}$ the groin point of the body. $V$ means the velocity vector of particle of the bottom, $n$ the time, $F$ the power vector of the particle and $F_{i}^{f}$ the friction of the particle $\mathrm{i}$. According equation 7 , the bottom is pulled up until its crotch collides with the groin point by the power of antigravity. Antigravity works as opposite direction to gravity, so it has minus value. Equation 8 represents the friction vector.

$$
f_{i}^{r}=-m_{i} G
$$


When the collision is detected a belt is set to the waist line which meets the top edge of the bottom. After setting the belt, the bottom is pulled down in order to stretching it since the bottom was slightly rolled up due to pulling up process. When the bottom is pulled up by the antigravity the force to a particle $i$ is shown in equation 9. After setting the belt the bottom is pulled down as shown in equation 10. Equation 9 and 10 has only one difference whether the antigravity $f_{i}^{r}$ is used or the gravity $f_{i}^{g}$ is done.

$$
\begin{aligned}
& F_{i}=f_{i}^{s}+f_{i}^{f}+f_{i}^{r}: \text { Before setting the belt } \\
& F_{i}=f_{i}^{s}+f_{i}^{f}+f_{i}^{g}: \text { After setting the belt }
\end{aligned}
$$

If the process for pulling down comes close a balance by interactions among the forces, the bottom can't be stretched no more. The finishing condition for fitting the bottom is introduced using the four points shown as reference points for finishing in figure 1 . Let $l_{1}$ be the difference between the reference points $e_{1}$ and $e_{2}$ in figure 1 , and $l_{2}$ be the difference between the reference points $e_{3}$ and $e_{4}$ in figure 1 , then the finishing condition for fitting can be represents in equation 11 . In the equation $l^{0}$ means the length before fitting and $l^{r}$ means the length after fitting, so $l_{1}^{0}$ represents the length $l_{1}$ before fitting.

$$
\begin{array}{ll}
I F\left(\left|\Delta d_{1}\right|+\left|\Delta d_{2}\right|<\epsilon \mid \epsilon \rightarrow 0\right) & )\left\{\begin{array}{cl}
Y e s, & V_{n+1}=0 \\
N o, & V_{n+1}=V_{n}+F_{n+1} d t / m
\end{array}\right. \\
\Delta d_{1}=l_{1}^{0}-l_{1}^{r}, \Delta d_{2}=l_{2}^{0}-l_{2}^{r} &
\end{array}
$$

Figure 4 shows the fitting process for a bottom whose lines across the body represent the stitching power vector between the front and back images of cloth.

For garment size, consumer can determine whether the garment fits or not in size by looking the fitting scene in the top. Most of the top such as T-shirt or one-piece do not lose the size information in the front and back images when it was folded. Whereas it can't be possible to decide the bottom in size-fitting since the folded bottom loses its part in the front and back images.
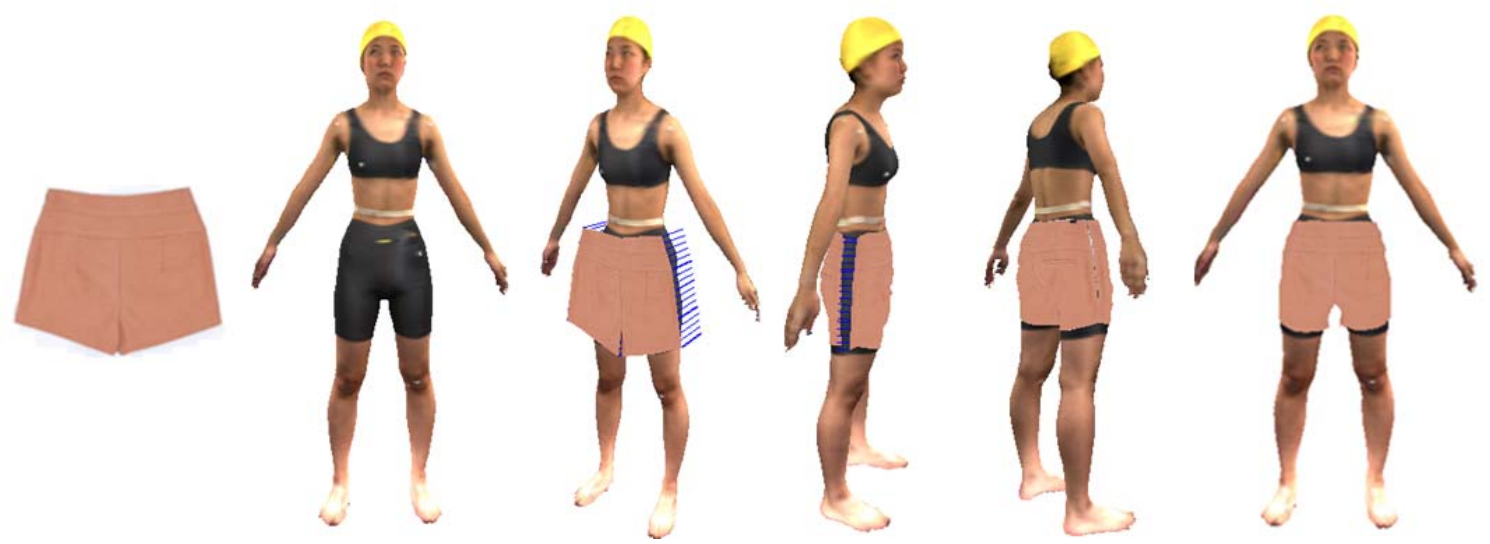

Fig. 4. The fitting process for the bottom

\subsubsection{Dressing in layers}

The processes reported in section 2.2.1 and 2.2.2 are for fitting only one cloth, but people in fact wear their garments in layers. Hence the fitting process for fitting in layers is reported in this section. For fitting in layers, the bottom firstly is fitted and the top later will be fitted. The flow chart in figure 5 shows the process. As seen in figure 5 the 3D body data should be loaded and reconstructed as the voxel data set. This reconstruction process is for the collision detection between the cloth image and the body. After the reconstruction the images of a garment are loaded. For the fitting in layers firstly the bottom should be fitted, so that the images of the bottom are loaded and fitted on the body data. If fitting the bottom was finished the coordinates of the body and the fitted cloth are integrated. The 
integrated data are newly reconstructed as a new voxel data set. The images of the top are fitted through the collision detection between the images and the new integrated body.

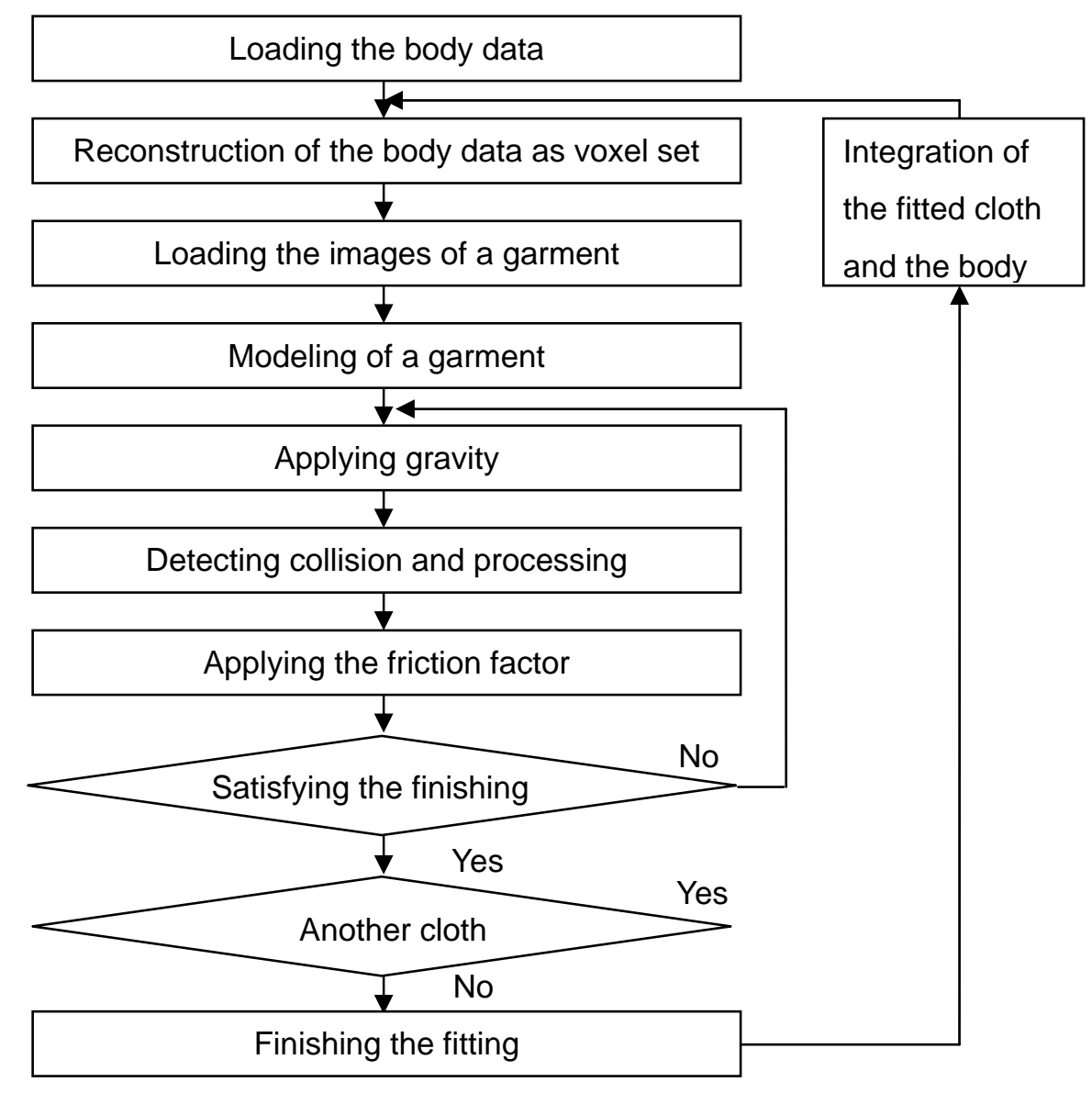

Fig. 5. Flow chart for fitting in layers

Figure 6 shows the fitting result in layers which fitted the top on the body wearing the bottom, bottom. The lines across the body represent the stitching power vector between the front and back images of cloth.
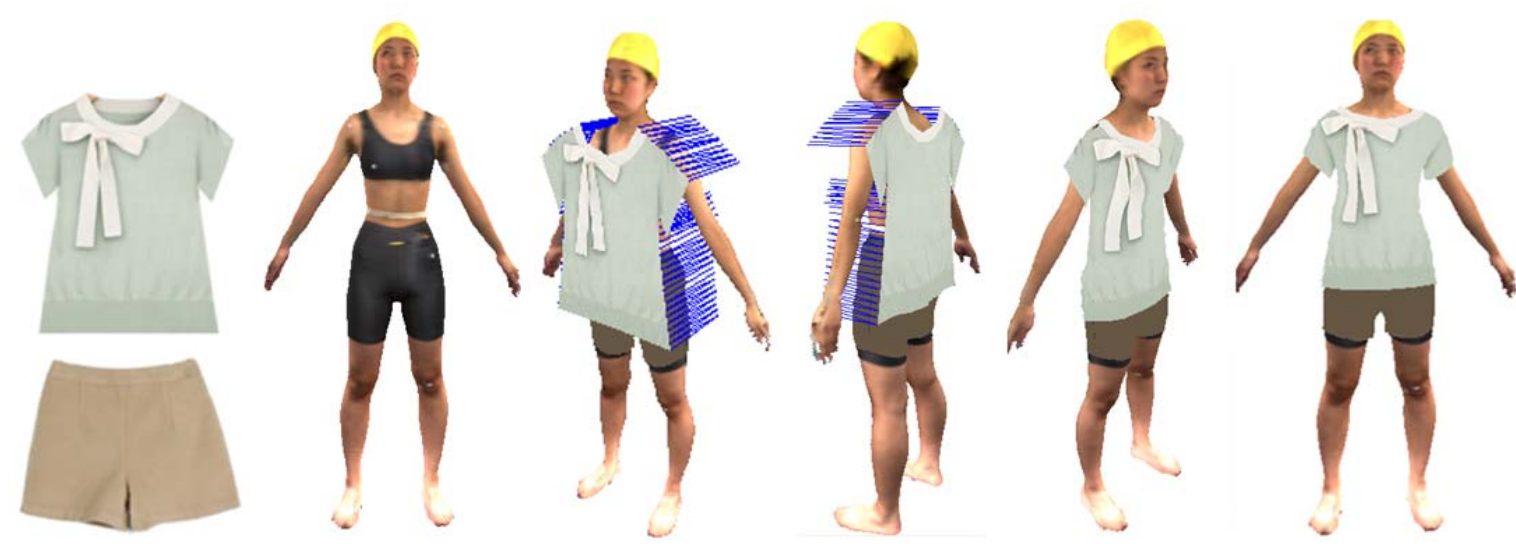

Fig. 6. shows the fitting result in layers which fitted the top on the body wearing the bottom. 


\section{Result}

The proposed garment-fitting system for merging the back and front images in our proposed system is shown to be effective. Also the fitting results for the top and bottom in layers shows it can be useful in the online market. Whereas there remains a problem to be enhanced in section 2.2.2, where the bottom can give the fitting result in appearance but can't give the fitting result in size.

As mentioned in introduction for the previous approaches using garment patterns, it is difficult for online retail shop to make garment patterns by itself. In contrast, we do not rely on pattern information, instead using only the front and back images of a garment. Our system is designed for a garment retail shop where simple garments are sold in a folded state on a rack. Some expensive garments having very complicated designs may require a system that uses pattern information, but our system can be applied to ordinary garments such as T-shirts, one-piece, skirts, pants, etc.

The computation needed for garment fitting can be carried out by individual users on their home PC, using the program with their personal body data stored locally. Retail shops would then exhibit and provide only the garment images in their website. Therefore, even customers who are concerned about the security of their body data can keep this data on their home PC only. In this way, personal data security will not be an issue. This may lead to a revolution in the retail garment market.

\section{References}

1. H.W.Seo, S.J.Kim, F.Cordier, K.H.Hong, (2007): "Validating a cloth simulator for measuring tight-fit clothing pressure", Proceedings of the ACM", pp.431-437

2. N.Magnenat-Thalmann, H.W.Seo, F.Cordier, (2004): "Automatic modeling of virtual humans and body clothing", Journal of Computer Science and Technology Vol.19, No.6, pp.575-584

3. F.Cordier, H.W.Seo, N.Magnenat-Thalman, (2003): "Made-to-measure technologies for an online clothing store", IEEE Computer Graphics and Application, pp.38-48

4. C.S.Cho, J.Y.Park, A.Boeing, P.Hington, (2010): "An implementation of a garment-fitting simulation system using laser scanned 3D body data", Computers in Industry, Vol.61, No.6, pp.550-558

5. S.W.Oh, (2002): "Interactive garment animation", Tech Memo 2002-1 KAIST (in Korean), pp.6-57

6. D.R.Haumann, (1987): "Modeling the physical behaviour of flexible objects", ACM SIGGRAPH' 87 Course Notes \#17-Topics in physically-based modeling

7. X.Provot,(1995): "Deformation constraints in a Mass-Spring Model to describe rigid cloth behavior", Proceedings of the Graphic Interface, pp.147-155

8. D.Baraff,(1998): "A. Witkin, Large steps in clothing simulation, in: Proceedings of the SIGGRAPH", pp.43-54 